\title{
Differential Recruitment of the Hippocampus, Medial Prefrontal Cortex, and the Human Motion Complex during Path Integration in Humans
}

\author{
Thomas Wolbers, ${ }^{1,2 \star}$ Jan M. Wiener, ${ }^{3,4 \star}$ Hanspeter A. Mallot, ${ }^{4}$ and Christian Büchel ${ }^{2}$ \\ ${ }^{1}$ Department of Psychology, University of California Santa Barbara, Santa Barbara, California 93106, ${ }^{2}$ Department of Systems Neuroscience, University \\ Medical Center Hamburg-Eppendorf, 20246 Hamburg, Germany, ${ }^{3}$ Laboratoire de Physiologie de la Perception et de l'Action, Collège de France, Centre \\ National de la Recherche Scientifique, 75231 Paris, France, and ${ }^{4}$ Department of Zoology, University of Tübingen, 72076 Tübingen, Germany
}

Path integration, the ability to sense self-motion for keeping track of changes in orientation and position, constitutes a fundamental mechanism of spatial navigation and a keystone for the development of cognitive maps. Whereas animal path integration is predominantly supported by the head-direction, grid, and place cell systems, the neural foundations are not well understood in humans. Here we used functional magnetic resonance imaging and a virtual rendition of a triangle completion paradigm to test whether human path integration recruits a cortical system similar to that of rodents and nonhuman primates. Participants traveled along two legs of a triangle before pointing toward the starting location. In accordance with animal models, stronger right hippocampal activation predicted more accurate updating of the starting location on a trial-by-trial basis. Moreover, between-subjects fluctuations in response consistency were negatively correlated with bilateral hippocampal and medial prefrontal activation, and bilateral recruitment of the human motion complex (hMT + ) covaried with individual path integration capability. Given that these effects were absent in a perceptual control task, the present study provides the first evidence that visual path integration is related to the dynamic interplay of self-motion processing in $\mathrm{hMT}+$, higher-level spatial processes in the hippocampus, and spatial working memory in medial prefrontal cortex.

Key words: path integration; navigation; virtual reality; spatial memory; hippocampus; functional MRI

\section{Introduction}

Path integration refers to monitoring self-motion to keep track of changes in orientation and position. It operates in a wide variety of species during the exploration of a new environment or in commuting between home and familiar resource sites. For example, ants and honeybees constantly update the position of the nest or hive (Collett and Collett, 2000), allowing them to return to their home base on a direct path after long winding excursions. By providing vector knowledge of locations occupied during outward journeys, path integration also constitutes a fundamental process for deriving cognitive maps from navigational experience.

Path integration relies on accurate perception of self-motion. Macaque medial superior temporal (MST) and ventral intraparietal (VIP) areas can extract heading information from optic flow, suggesting a role in self-motion analyses based on visual cues (Bremmer et al., 2002; Duffy et al., 2005). Head-direction

Received Dec. 21, 2006; revised July 12, 2007; accepted July 13, 2007.

This work was supported by the European Commission, Marie Curie Outgoing International Fellowship (T.W.) the Volkswagenstiftung, the German Ministry of Education and Research (Bundesministerium für Bildung und Forschung), and the German Research Foundation (Deutsche Forschungsgemeinschaft MA 1038/9-1, WI 2729/1-1).

We thank Jack Loomis and Eszter Schoell for their helpful comments on previous drafts of this manuscript.

${ }^{*}$ T.W. and J.M.W. contributed equally to this work.

Correspondence should be addressed to Thomas Wolbers, Department of Psychology, University of California

Santa Barbara, Santa Barbara, CA 93106. E-mail: wolbers@psych.ucsb.edu.

DOI:10.1523/JNEUROSCI.2146-07.2007

Copyright $\odot 2007$ Society for Neuroscience $\quad 0270-6474 / 07 / 279408-09 \$ 15.00 / 0$ cells in retrosplenial cortex are also sensitive to visual stimulation (Vann and Aggleton, 2004), and this directional information affects hippocampal place cells that code for an animal's location independent of heading (Etienne and Jeffery, 2004). Place cells are further modulated by running speed (O’Keefe et al., 1998), indicating that they have access to direction and distance information, which would foster the establishment of place fields and the computation of homing vectors (but see Alyan and McNaughton, 1999). However, the recent discovery of entorhinal grid cells suggests that pivotal spatial information for path integration might already be computed upstream of the hippocampus (Hafting et al., 2005). Ultimately, intimate connections between the hippocampus and subdivisions of the prefrontal cortex (Ferino et al., 1987; Barbas and Blatt, 1995) appear to be a neuronal mechanism for holding the integrated spatial signal in working memory (Lee and Kesner, 2003; Jones and Wilson, 2005). For example, the correlation between hippocampal and medial prefrontal firing is selectively enhanced when rats use spatial information about a starting location to guide their behavior (Jones and Wilson, 2005), suggesting that prefrontal engagement complements the hippocampal contribution to path integration.

The present study provides the first attempt to characterize the cortical network that supports visual path integration in humans. We used functional magnetic resonance imaging (fMRI) and a virtual environment to test whether human path integra- 

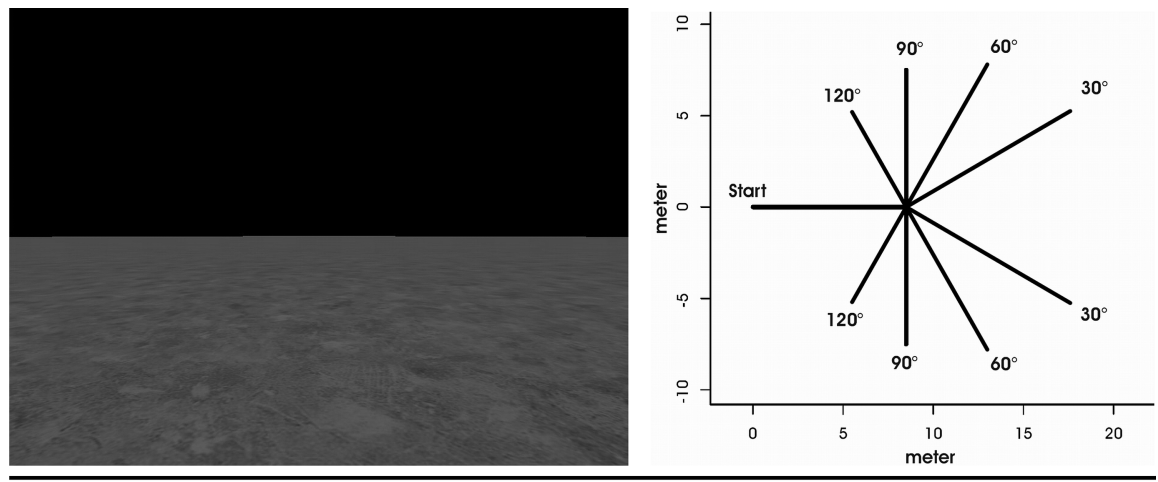

ABSOLUTE POINTING ERRORS


Figure 1. Experimental stimuli, absolute pointing errors, and reaction times (mean \pm SEM). Top left, Subjects experienced passive self-motion through a virtual environment from a first-person perspective. During both path integration and control trials, participants were passively transported along two legs of a triangle. Optic flow arising from a ground texture was the only available cue to determine speed and direction of self-motion. On reaching the endpoint of the second translation, subjects were requested to point with an MR-compatible joystick either to the starting location (path integration trials) or in the egocentric direction of an arrow presented at the beginning of each trial (control trials). Top right, Layout of the eight triangles that comprised two translations and one intermediate rotation. The length of the first translation was kept constant across trials to ensure identical onsets of the intermediate rotation. Rotations differed in turning direction (left vs right) and turning angle $\left(30^{\circ}, 60^{\circ}, 90^{\circ}\right.$ and $120^{\circ}$ ). We adapted the lengths of the second translation to further guarantee that total travel duration did not differ between trial types. Specifically, the length of the second leg was shorter for trials with longer rotations and vice versa. Note that we used identical path layouts for path integration and control trials. In addition, required pointing angles were matched between path integration and control trials. Bottom, Absolute pointing errors (left) were below $20^{\circ}$ for all trial types, suggesting that participants could effectively use optic flow to update the starting location. Statistical analysis of absolute pointing errors and reaction times (right) revealed that path integration and control trials did not differ significantly with respect to task difficulty. Values on the $x$-axes indicate the required pointing responses relative to the observer's final heading.

tion recruits a cortical system similar to that of rodents and nonhuman primates. Subjects traveled along two legs of a triangle before pointing toward the starting location with a joystick. Simulated motion was passive to ensure identical travel duration across subjects and to avoid confounding motor activation (caused by joystick manipulation). During control trials, while traveling along paths identical to those used for the path integration trials, subjects memorized the egocentric direction of an arrow (i.e., $90^{\circ}$ to the right) presented before each trial. On reaching the endpoint of the second leg, participants reproduced the pointing direction of the arrow in an egocentric reference frame, i.e., without taking into account the change in heading they had experienced during the outward journey. To accurately identify the critical structures in the brain, we integrated behavioral performance in the analyses of the fMRI data.

\section{Materials and Methods}

Subjects. Sixteen healthy volunteers with normal or corrected-to-normal vision gave written informed consent to participate in this study, which was approved by the local ethics committee. All subjects understood the instructions without difficulties, and none were aware of the hypotheses at the time of testing. Because three participants had to be removed as a result of corrupt behavioral or neuroimaging data (see Results), the final dataset comprised 13 subjects (age range, 21-29 years; seven men).

Experimental stimuli. We used the Virtual Environments Library (www.kyb.mpg.de/prjs/ facilities/velib) to animate a desktop virtual environment (Fig. 1) that participants viewed from a first-person perspective (eye height, 160 $\mathrm{cm})$. Optic flow information was only provided by a floor texture, and the remainder of the scene was kept black. For the experimental and control tasks that were administered during fMRI scanning, we designed a set of eight outbound paths constituting two legs of a triangle (Fig. 1). Each path comprised two translations and one intermediate rotation, and the length of the first translation was kept constant across trial types $(8.5 \mathrm{~m})$. This ensured identical onsets of the intermediate rotation that differed in turning direction (left vs right) and turning angle $\left(30^{\circ}, 60^{\circ}, 90^{\circ}\right.$, and $\left.120^{\circ}\right)$. Given that path integration in virtual environments is most accurate when displacement velocity resembles natural locomotion (Ellmore and McNaughton, 2004), we adopted a speed of a moderately paced walk. Translations (maximum speed, 2 $\mathrm{m} / \mathrm{s}$ ) and rotations (maximum speed, $40 \% \mathrm{~s}$ ) were performed successively and followed the same trapezoid velocity profile with linear velocity increases and decreases. The duration of the plateau of the trapezoid changed according to the length of the translation or rotation angle, respectively. In addition, we adapted the lengths of the second translation to further guarantee that the total travel duration did not differ between trial types. Specifically, the length of the second leg was shorter for trials with longer rotations and vice versa.

For training sessions, we designed a different set of eight triangles. While the length of the first translation was kept constant at $8.5 \mathrm{~m}$, we used turning angles of $45^{\circ}, 75^{\circ}, 105^{\circ}$, and $135^{\circ}$. In addition, the length of the second translation also differed between training and fMRI trials, given that it was always adjusted to ensure a constant travel duration of $11.9 \mathrm{~s}$. Because of these differences in path layout, none of the pointing responses required during training sessions matched those required in the fMRI sessions.

Procedure. All participants first completed two training sessions, followed by one experimental session and a final control session. During experimental sessions, subjects were passively transported along two legs of a given triangle. Each trial started with a static presentation of the virtual environment (duration, $4 \mathrm{~s}$ ), followed by the outward journey that always lasted $11.9 \mathrm{~s}$. When the endpoint of the second translation was reached, participants used an MR-compatible joystick (Mag Design and Engineering, Sunnyvale, CA) to point toward the origin of travel within a $5 \mathrm{~s}$ interval. Pointing responses were automatically recorded when joystick deflection exceeded a virtual circle corresponding to $80 \%$ of maximal deflection. A black screen was presented during intertrial intervals that randomly varied between 4 and $5 \mathrm{~s}$.

Before the experimental session, we performed extensive training to eliminate learning and habituation effects. Detailed instructions about the task were followed by a real-world version outside the MR environment: after a guided walk along two legs of a randomly chosen triangle, 
subjects had to point toward the starting location using the joystick that was also used for the virtual environments. Next, participants completed 16 training trials presented on a standard computer monitor (two replications of each training path; see above, Experimental stimuli). Only during training trials were pointing responses followed by instant feedback: an arrow was presented that indicated the correct pointing direction. In case a participant failed to update heading as reflected by a confusion of pointing directions (i.e., leftward instead of rightward pointing after a rightward rotation), the real-world task was replicated once. This procedure proved successful because all participants were subsequently able to perform the task with relatively low pointing errors (see Results). After being positioned within the bore of the magnet, subjects were given another set of 16 training trials without concurrent fMRI recording to familiarize them with performing the task while being in a horizontal position. In the subsequent experimental session, five replications of each path were presented in pseudorandomized order, giving a total of 40 trials. However, participants were not informed about trial repetitions, and none of these trials provided behavioral feedback. All trials were scanned in one fMRI session.

Visual path integration does not only involve the processing of selfmotion cues during the outbound path, but it also encompasses a working memory component for changes in distance and direction from the starting point. Therefore, we designed a control task that provided identical visual stimulation during the outbound path while involving a working memory component unrelated to that path. In the final control session, subjects were confronted with the same virtual environment and the same 40 paths as in the experimental session. However, an arrow, aligned parallel to the ground plane, was presented during the initial static phase of each trial (duration, $4 \mathrm{~s}$ ). Subjects were instructed to memorize its pointing direction relative to their initial heading (i.e., $90^{\circ}$ to the right) and then to pay attention to the outward journey. After reaching the endpoint of the second translation, participants had to point in the direction previously indicated by the arrow within a $5 \mathrm{~s}$ interval. Specifically, subjects were required to reproduce the pointing direction of the arrow in an egocentric reference frame (i.e., $90^{\circ}$ to the right), without taking into account the change in heading they had experienced during the outbound path. Consequently, the control task did not require subjects to path integrate, because the correct response, as specified by the pointing direction of the arrow, was independent of the characteristics of the outbound path.

Although we deliberately scanned path integration and control tasks in separate sessions to minimize the possibility that subjects engaged in path integration during the control task, it remains possible that subjects automatically updated the position of the starting location. In case the required pointing response (as specified by the arrow) differed from the direction toward the starting location, this could potentially elicit a mismatch-related signal. To minimize a potential influence of this confounding effect, we ensured that, in the control condition, the pointing direction of the arrow always coincided with the relative direction toward the origin of travel. Furthermore, this design allowed us to completely match the distribution of the required pointing responses between experimental and control trials. As for the experimental session, intertrial intervals randomly varied between 4 and $5 \mathrm{~s}$, and all trials were scanned in one fMRI session. In summary, control trials provided identical visual stimulation, involved a working memory component unrelated to the outbound path, and did not require subjects to path integrate.

MRI acquisition. MR scanning was performed on a 3T MRI Scanner (Trio; Siemens, Munich, Germany) with a standard head coil. Thirtyseven contiguous axial slices (slice thickness, $3 \mathrm{~mm}$ ) were acquired using a gradient echo echo-planar $\mathrm{T} 22^{\star}$-sensitive sequence (repetition time, $2.12 \mathrm{~s}$; echo time, $25 \mathrm{~ms}$; flip angle, $70^{\circ}$; matrix, $64 \times 64$; field of view, $192 \times 192 \mathrm{~mm}$ ). A liquid crystal display projector back-projected the virtual environment on a screen positioned on top of the head coil. Subjects lay on their backs within the bore of the magnet and viewed the stimuli comfortably via a $45^{\circ}$ mirror that reflected the images displayed on the screen. To minimize head movement, all subjects were stabilized with tightly packed foam padding surrounding the head.

Statistical analysis of behavioral data. Direction judgments of left-turn paths were mirrored, allowing us to collapse pointing responses and pointing errors across left and right turns. Because of the directional nature of pointing data, we used circular statistics to analyze pointing performance. In contrast, standard statistical measures were computed for reaction time data. Two-way ANOVA with task and turning angle as within-group factors served to elucidate differences between experimental and control sessions.

Image processing and statistical analysis of $f M R I$ data. Image processing and statistical analysis were performed using SPM2 (Wellcome Department of Imaging Neuroscience, UCL, London, UK). All volumes were realigned to the first volume, spatially normalized to an echo planar imaging template in a standard coordinate system (Evans et al., 1993), and finally smoothed using a $9 \mathrm{~mm}$ full-width at half-maximum isotropic Gaussian kernel. At the single-subject level, we applied a high-pass filter to remove baseline drifts. Design matrices containing two separate sessions (experimental and control) were specified, thereby removing session-specific effects. Outbound paths and pointing responses were modeled as boxcar functions convolved with a hemodynamic response function (HRF). The predicted responses covered the entire outward journeys and the entire response intervals, respectively. Trials in which participants failed to respond within the $5 \mathrm{~s}$ interval were modeled as separate regressors and were discarded during statistical analyses. We specified separate regressors for each turning angle $\left(30^{\circ}, 60^{\circ}, 90^{\circ}\right.$, and $\left.120^{\circ}\right)$, collapsing across right and left turns. To assess within-subjects performance effects on a trial-by-trial basis, we added regressors in which the HRFs of the path regressors, for both the experimental and the control condition, were parametrically modulated with absolute pointing error on each trial. In addition, we created two additional models to test for an overall habituation of the blood oxygenation level-dependent (BOLD) response across sessions. Specifically, we created additional regressors in which the HRFs of the path regressors for both conditions were parametrically modulated with (1) the time elapsed since the onset of the experiment or (2) the repetition of trials (i.e., first occurrence, second occurrence, etc.). Most importantly, these regressors covered the entire experiment, allowing us to assess overall habituation effects across both sessions. Specific effects were tested with appropriate linear contrasts of the parameter estimates, and the corresponding contrast images were subsequently entered into a random effects analysis. Main effects of path integration, performance-related activation, and habituation effects were assessed with one-sample $t$ tests.

In addition to examining the effects of within-subjects performance variability, we also investigated the relationship between BOLD responses and between-subjects variability. Pointing errors in a path integration paradigm reflect a compound of different error sources. An appropriate analysis of between-subjects performance effects therefore requires a decomposition of pointing errors. Overall bias, the general tendency to overshoot or undershoot, was quantified as the circular mean of the signed pointing errors. Because humans tend to produce variable responses for repetitions of a given path, we also computed random error, reflecting fluctuations in response consistency, as the average circular SD of the signed pointing error. And finally, we fitted a linear regression model to the pointing data of each participant to capture systematic error. The slope of the regression line is thought to reflect the individual potential to encode information about the pathway and to appreciate differences between paths (Fujita et al., 1993). Subjects who are unable to precisely encode self-motion information often show a tendency to produce stereotyped responses: an overshooting for small angles and an undershooting for large angles. Because an ideal regression line would have a slope of 1 , we quantified systematic error as the absolute difference between the individual slope and the ideal value of 1 . To identify the neural foundations of between-subjects variability, we entered individual contrast images reflecting mean activation during encoding of the outbound path into a multiple regression analysis (one image per participant). Values for overall bias along with random and systematic error scores served as predictor variables, allowing for the identification of the unique contribution of each error source. To test for the specificity of any potential results, we analyzed both within- and between-subjects performance effects not only for the path integration task but also for the control task.

For all analyses, we used an extent threshold of five contiguous voxels 


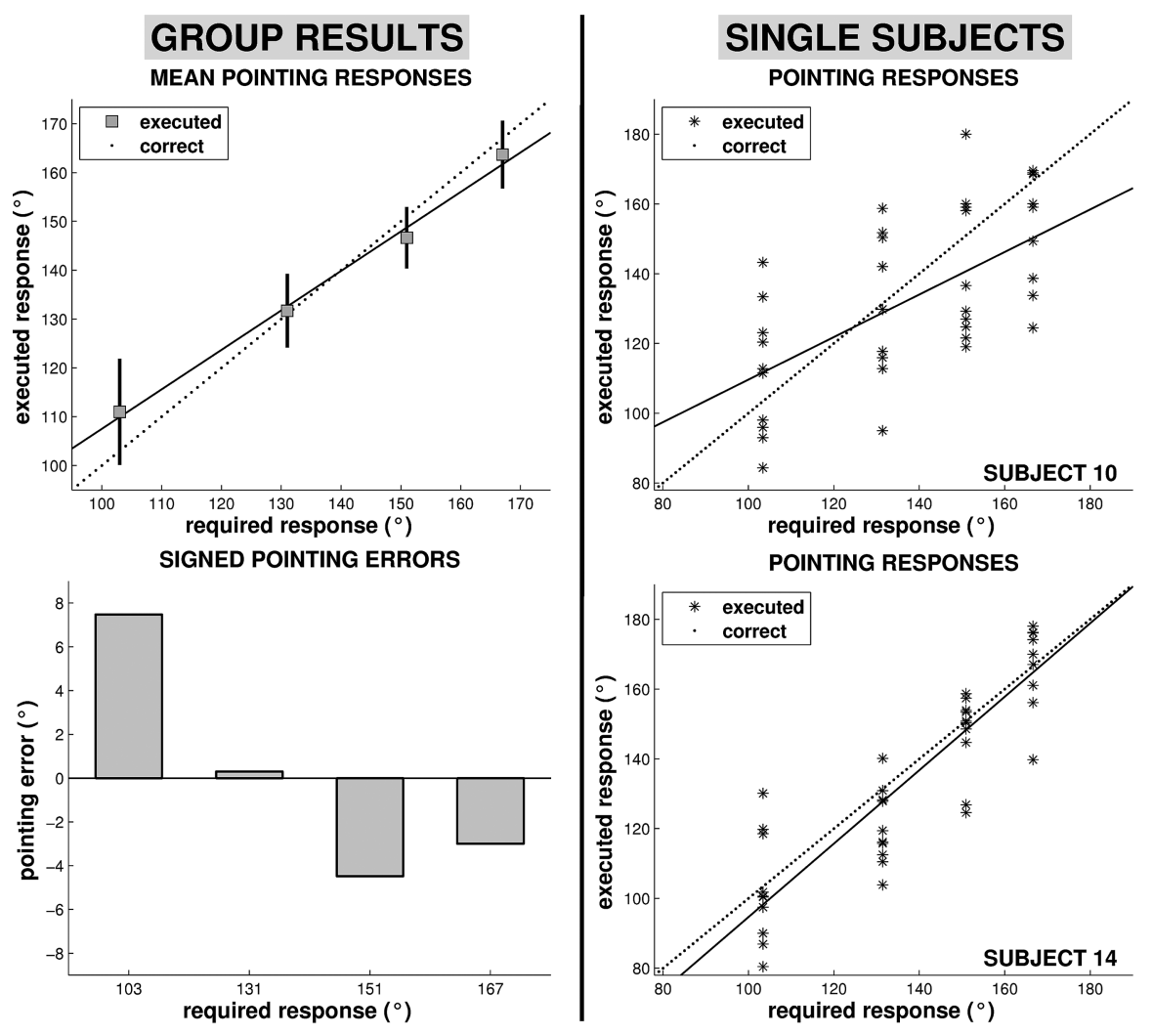

Figure 2. Pointing performance and signed pointing errors. Left, Average pointing performance (mean \pm SD) and signed pointing errors for the path integration task revealed an overall bias to overshoot small and to undershoot large required pointing angles. Uncertainties about the magnitude of translations and rotations result in a tendency to adopt prototypical values. Most importantly, error bars demonstrate substantial interindividual variability, suggesting that participants differed in path integration capability. Right, Single-trial pointing performance for two individuals that differed substantially in both random and systematic error. Subject 14 shows less pointing variability for each trial type, indicating a more consistent task engagement compared with subject 10. The greater tendency to produce prototypical responses in subject 10 suggests problems with appreciating differences between path layouts. These differences in path integration potential are well captured by the different slopes of the regression lines. For all panels, values on the $x$-axes indicate the required pointing responses relative to the observer's fina heading.

and a voxel-level height threshold of $p<0.05$ (familywise error rate) to correct the resulting statistical parametric maps for multiple comparisons. To take into account our a priori hypotheses, we defined anatomical regions of interest (ROIs) for the hippocampus, entorhinal cortex, medial prefrontal cortex (mPFC), MST, VIP, and the retrosplenial cortex. Whenever possible, we created anatomical regions of interest (comprising entire structures) to correct for multiple comparisons. The Wake Forest University Pickatlas (Maldjian et al., 2003) and the anatomy toolbox of SPM (Eickhoff et al., 2005) served to define the hippocampus, the entorhinal cortex, and the superior medial frontal gyrus. However, we are not aware of any precise definition of regions MST, VIP, and retrosplenial cortex in humans. Therefore, for these regions, we created spherical volumes with a $12 \mathrm{~mm}$ radius surrounding previously reported coordinates [shown throughout text as $(x, y, z)]$ : MST, $( \pm 45,-60,5)$ (Dukelow et al., 2001); VIP, ( $\pm 38,-44,46)$ (Bremmer et al., 2001); retrosplenial cortex, $( \pm 4,-62,24)$ (Wolbers and Büchel, 2005). In all ROIs, correction for multiple comparisons was based on the respective ROI; elsewhere in the brain, it was based on the entire search volume.

\section{Results}

\section{Behavioral performance}

Three participants were excluded from the final dataset: one subject showed excessive head movement, leading to missing data in a large portion of the occipital cortex. Two other participants had very large pointing errors in the control task, exceeding the group means by more than 2 SDs. Mean reaction times of the remaining
13 participants were below $1600 \mathrm{~ms}$ (Fig. 1). A $2 \times 4$ repeated-measures ANOVA [factors of task (experimental vs control) and turning angle $\left.\left(30-120^{\circ}\right)\right]$ revealed neither significant main effects of turning angle $(F=1.692, p=0.204)$ and task $(F=$ 4.106; $p=0.066)$ nor an interaction between both factors $(F=0.331 ; p=0.742)$.

Mean absolute pointing errors were below $20^{\circ}$ for all route types (Fig. 1) and were similarly affected by our experimental manipulations: main effect of turning angle, $F=0.308, p=0.752$; main effect of task, $F=3.761, p=0.076$; interaction, $F=$ 1.931, $p=0.173$. To further explore the trend for a significant main effect of task, we performed pairwise planned contrasts between path integration and control conditions. This analysis revealed the trend for an overall difference to be predominantly driven by the difference in the $103^{\circ}$ condition $\left(103^{\circ}, p=0.037 ; 131^{\circ}, p=0.227\right.$; $\left.151^{\circ}, p=0.751 ; 167^{\circ}, p=0.727\right)$. Most importantly, when analyzing pointing responses in the $103^{\circ}$ condition, we observed that, across replications of trials, the average SD of pointing responses was much higher for the path integration than the control trials (13.42 vs 6.66; $p<0.001$ ). Given that absolute pointing errors represent the absolute difference between required and executed pointing responses, a larger variability around the correct value therefore inflated mean absolute pointing error.

To test for systematic changes in pointing performance over the course of the experimental or the control session, we computed the correlation between absolute pointing error and trial number for each participant, transformed those correlations to normally distributed variables using Fisher's $z$-transformation, and then computed $t$ tests on the transformed correlations. In both conditions, $<2 \%$ of the performance variability was related to increasing familiarity with the traveled paths (experimental, $r=-0.11, p=0.08$; control, $r=$ $-0.07, p=0.19)$. These results indicate that the extensive pretraining (see Materials and Methods) successfully prevented learning and habituation effects from occurring over the course of the experiment.

Pointing performance in the path integration task showed an overall tendency to overshoot small and to undershoot large required pointing angles (Fig. 2). A one-way ANOVA on the signed pointing errors confirmed a significant effect of turning angle $(F=9.40 ; p<0.001)$. Most importantly, the SDs of the mean pointing responses demonstrate significant between-subjects variability, which is further illustrated by the single-subject data. The two participants depicted in Figure 2 differ substantially in both random and systematic error. On the one hand, subject 14 shows significantly less pointing variability for each trial type, presumably indicating a more consistent task engagement compared with subject 10 . On the other hand, the greater tendency to produce prototypical responses in subject 10 suggests problems with appreciating differences between path layouts. These differ- 
ences in path integration capability are well captured by the different slopes of the regression lines.

\section{fMRI results}

In each of the following subsections, we present the results from analyzing BOLD responses during the outward journey in the entire brain. As outlined in Materials and Methods, we took into account our neuroanatomical a priori hypotheses by defining regions of interest for structures we predicted to be involved in visual path integration.

Main effect of path integration

We first contrasted path integration with the control condition to identify overall activation attributable to attending to and processing of self-motion cues. This analysis yielded several significant activations, including the precuneus, subdivisions of the intraparietal sulcus, the posterior middle temporal gyrus, and several frontal areas. Table 1 lists the significantly activated areas according to Montreal Neurological Institute (MNI) space (Evans et al., 1993) along with coordinates and statistical results for the respective peak voxels.

\section{Within-subjects performance effects}

The overall comparison between path integration and the control condition presumably identified regions that are not only implicated in self-motion processing but also in attention to motion in general. To determine areas specifically involved in visual path integration, we included absolute pointing errors, a measure of trial-bytrial accuracy, as a parametric modulation regressor in the general linear model. Increased activation during encoding of the outbound path was associated with more accurate pointing performance in the right hippocampus only (Fig. 3, Table 2). This effect was independent of general performance levels: for both good and bad path integrators, we observed a negative correlation between absolute pointing error and engagement of the right hippocampus. Elsewhere in the brain, we did not obtain any significant results. Given that the different sizes of the predefined ROIs can induce a bias in the form of different statistical thresholds, we also analyzed the parametric modulation using an uncorrected threshold of $p<0.001$. This analysis did not reveal any significant voxels outside the hippocampus, in neither the remaining ROIs nor the rest of the brain, thus lending additional support to the specificity of the results.

An identical analysis on the control condition, using absolute pointing errors in the control task as a measure of trial-by-trial accuracy, did not yield any significant effects, in neither the right
Table 1. Path integration versus control

\begin{tabular}{|c|c|c|c|c|}
\hline \multirow[b]{2}{*}{ Region } & \multicolumn{2}{|c|}{ Coordinates $(x, y, z$, in mm) } & \multirow{2}{*}{$\begin{array}{l}\text { Voxel level } \\
\text { (z-score) }\end{array}$} & \multirow{2}{*}{$\begin{array}{l}\text { Cluster size } \\
\text { (\# of voxels) }\end{array}$} \\
\hline & LH & RH & & \\
\hline \multirow[t]{3}{*}{ Inferior occipital gyrus } & & $36,-90,0$ & 5.97 & 125 \\
\hline & $-36,-76,-12$ & & 5.68 & 118 \\
\hline & $-32,-90,-4$ & & 4.93 & \\
\hline Inferior temporal gyrus & & $46,-54,-18$ & 5.87 & 238 \\
\hline Fusiform gyrus & & $40,-60,-18$ & 5.69 & \\
\hline \multirow[t]{2}{*}{ Middle temporal gyrus complex } & & $50,-58,6$ & 4.87 & 554 \\
\hline & $-42,-66,6$ & & 4.30 & 347 \\
\hline \multirow[t]{2}{*}{ Precuneus } & & $26,-62,32$ & 5.68 & 35 \\
\hline & $-22,-66,34$ & & 5.43 & 23 \\
\hline \multirow[t]{2}{*}{ Posterior intraparietal sulcus } & & $32,-56,48$ & 5.39 & 20 \\
\hline & $-30,-62,52$ & & 4.52 (NS) & \\
\hline \multirow[t]{2}{*}{ Anterior intraparietal sulcus } & $-42,-36,50$ & & 5.72 & 84 \\
\hline & & $46,-34,48$ & 4.61 (NS) & \\
\hline Superior frontal gyrus & & $28,4,68$ & 5.12 & 10 \\
\hline \multirow[t]{2}{*}{ Superior medial frontal gyrus } & $4,24,50$ & & 4.04 & 63 \\
\hline & & $-4,24,40$ & 4.78 & 170 \\
\hline
\end{tabular}

Spatial coordinates of the local maxima in the group analysis ( $p<0.05$ corrected). RH, Right hemisphere; LH, left hemisphere; NS, not significant.

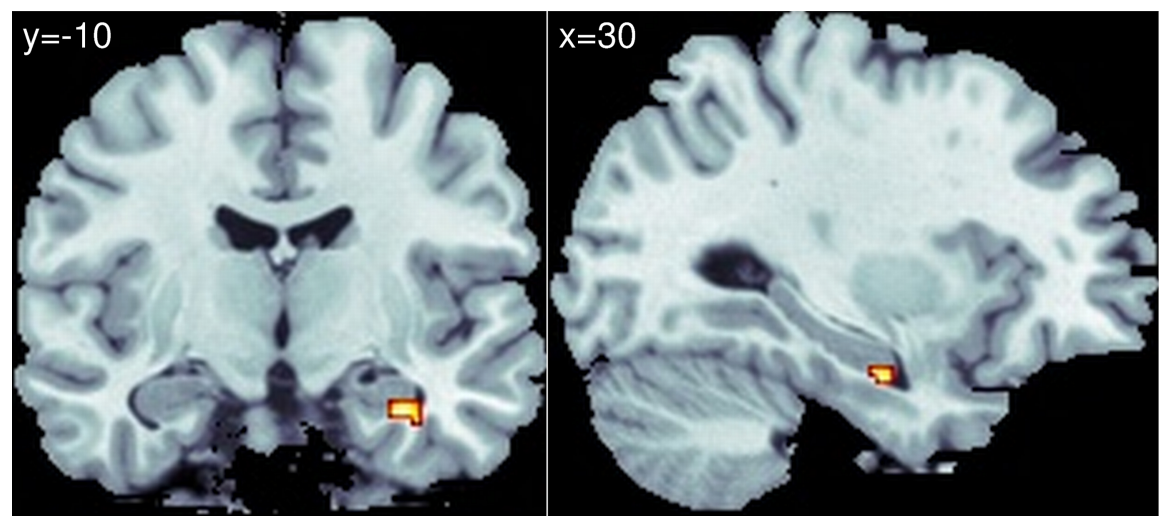

Figure 3. Within-subjects performance effects. Parametric analysis revealed a tight correspondence between right hippocampal activation during encoding of the outbound path and absolute pointing error on a trial-by-trial basis. Stronger engagement of the right hippocampus was associated with more accurate pointing performance in both good and bad path integrators. Performing the same analyses with the control task did not reveal any significant effects. To show the subthreshold extent of the activated region, results of the random effects analysis are displayed with a threshold of $p<0.01$ (uncorrected) on the MNI template brain.

\section{Table 2. Performance-related effects}

\begin{tabular}{|c|c|c|c|c|}
\hline \multirow[b]{2}{*}{ Region } & \multicolumn{2}{|c|}{ Coordinates $(x, y, z$, in mm) } & \multirow{2}{*}{$\begin{array}{l}\text { Voxel level } \\
\text { (z-score) }\end{array}$} & \multirow{2}{*}{$\begin{array}{l}\text { Cluster size } \\
\text { (\# of voxels) }\end{array}$} \\
\hline & $\mathrm{LH}$ & $\mathrm{RH}$ & & \\
\hline \multicolumn{5}{|l|}{ Within-subjects performance effects } \\
\hline Hippocampus & & $30,-10,-24$ & 3.54 & 5 \\
\hline \multicolumn{5}{|l|}{ Between-subjects performance effects } \\
\hline \multicolumn{5}{|l|}{ Random error } \\
\hline \multirow[t]{2}{*}{ Hippocampus } & & $24,-10,-20$ & 3.52 & 23 \\
\hline & $-24,-14,-22$ & & 3.29 (NS) & \\
\hline \multirow{3}{*}{ Superior medial frontal gyrus } & & $10,58,12$ & 3.90 & 90 \\
\hline & & $12,46,46$ & 3.78 & \\
\hline & $-12,44,40$ & & 3.82 & 19 \\
\hline \multicolumn{5}{|l|}{ Systematic error } \\
\hline \multirow[t]{2}{*}{ hMT+ } & & $50,-66,12$ & 4.18 & 10 \\
\hline & $-46,-66,16$ & & 3.55 & 5 \\
\hline
\end{tabular}

Spatial coordinates of the local maxima in the group analysis ( $p<0.05$ corrected). RH, Right hemisphere; LH, left hemisphere; NS, not significant.

hippocampus [i.e., $p=0.63$ at $(30,-10,-24) \Rightarrow$ hippocampal peak voxel identified in the experimental condition] nor elsewhere in the brain. Finally, we tested whether the right hippocampal cluster was not only absent in the control condition but whether it was also significantly stronger correlated with performance for the path integration than the control trials. A paired $t$ 


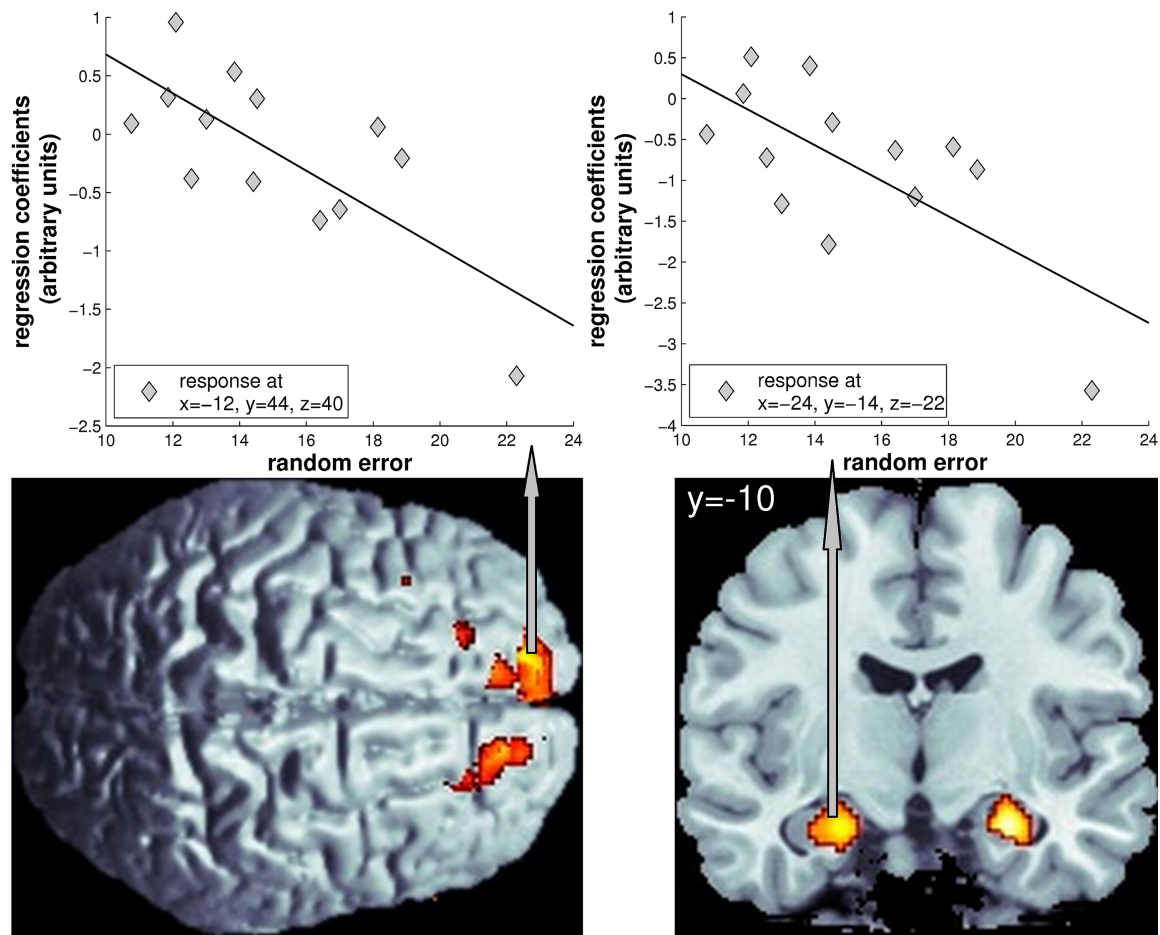

Figure 4. Between-subjects performance effects: random error. Random error was negatively correlated with hippocampal and medial prefrontal activation during encoding of the outbound path across subjects. In both regions, overall activation was greater in those participants who showed higher response consistency. Top displays individual regression coefficients of the respective peak voxels along with average SDs of the signed pointing error ( $\Rightarrow$ random error). Semipartial correlation coefficients of the peak voxels ranged from $r=-0.80$ [at coordinates $(-24,-14,-22)$ ] to $r=-0.82$ [at coordinates $(-12,44,40)$ ]. Elsewhere in the brain, we did not observe any significant results. Performing the same analyses on the control data did not revea any significant effects in the hippocampus; however, we observed a correspondence between random error and activation in the left medial prefrontal cortex. To show the subthreshold extent of the activated regions, bottom displays the results of the random effects analysis with a threshold of $p<0.01$ (uncorrected) on the MNI template brain.

test first identified voxels showing a stronger parametric modulation for the path integration than the control condition. The resulting statistical image was then applied as an explicit mask to a reanalysis of the parametric modulation for the path integration condition. This analysis yielded the same cluster in the right hippocampus compared with the initial analysis.

Between-subjects performance effects

To elucidate the neural mechanisms that differentiated good from bad path integrators, we decomposed each participant's pointing errors into (1) overall bias, the general tendency overshoot or undershoot, (2) random error, reflecting fluctuations in response consistency, and (3) systematic error, pertaining to the individual potential to encode information about the pathway (for details, see Materials and Methods). A multiple regression served to identify areas in which activation covaried significantly with each error type across subjects.

While we did not observe any significant effects of overall bias, random error was negatively correlated with bilateral responses in the hippocampus and the medial prefrontal cortex (Fig. 4, Table 2). Although activation in the left hippocampal peak voxel did not withstand a multiple comparisons correction for the entire hippocampus ( $p=0.085$ ), the extent of the activated cluster and the close association with random error scores both suggest a substantial contribution. In all these regions, overall activation during path integration was greater in those participants who showed higher response consistency. Elsewhere in the brain, we did not obtain any significant results. As for the within-subjects variability, we also looked at each ROI when applying an uncorrected threshold of $p<0.001$, but this analysis only confirmed the bilateral effects in the hippocampus and the medial prefrontal cortex. Outside of these ROIs, negative correlations at $p<0.001$ uncorrected were observed bilaterally in the inferior frontal and the middle temporal gyri and in the left rectal gyrus. However, because these regions were not included in our a priori hypotheses, these results should be considered exploratory.

Systematic error was closely related to bilateral activation in the posterior part of the middle temporal gyrus (Fig. 5, Table 2 ). This area exhibited a positive correlation: participants who were able to encode self-motion information very precisely showed weaker responses than participants with large systematic error scores. Given that the effect was located in the region of interest that was defined using previously reported coordinates for area MST (see Materials and Methods), it is reasonable to assume that the activated cluster encompassed the human homolog of monkey area MST (Dukelow et al., 2001; Huk et al., 2002). However, we cannot exclude the possibility that area MT and adjacent satellite areas of the human motion complex $(\mathrm{hMT}+)$ may also have been recruited. When applying an uncorrected threshold of $p<0.001$, we obtained two additional clusters in the anterior part of the left middle temporal gyrus. Again, given that this region was not included in our a priori hypotheses, these results should be considered exploratory. Testing for a negative correlation did not reveal any significant results.

In the control condition, we did not observe significant effects of overall bias and systematic error. In a similar vein, the amount of random error was not significantly correlated with responses anywhere in the brain, the exception being the left medial prefrontal cortex $[p=0.035$ at $(-10,40,56)]$. In analogy to the parametric modulation analysis, we reran each analysis for the path integration condition but excluded voxels that failed to show a significantly stronger correlation with performance for the path integration than the control trials. The results confirmed the effects observed for both random (hippocampus and medial prefrontal cortex) and systematic (middle temporal gyrus) error, thus indicating that the correspondences between BOLD responses and pointing performance were specific to the path integration condition.

\section{Overall decay of activation}

Given that path integration and control sessions were always presented in a fixed order (see Materials and Methods), any differences between both conditions could theoretically be related to an overall habituation of the BOLD signal. Therefore, we performed an additional analysis to test whether the repetition of the outbound paths led to decreasing responses across sessions. Furthermore, because the order of trials was randomized across subjects, we also modeled a habituation of the BOLD signal with 
respect to the time elapsed since the onset of the experiment. While we observed an overall decay of activation in the right supplementary motor area only with respect to time, both analyses revealed significant effects in the inferior parietal lobe bilaterally (Table 3). However, we did not obtain significant results in any of the areas identified by the performance-related analyses.

\section{Discussion}

Visual path integration recruited a cortical system similar to that of rodents and nonhuman primates. Right hippocampal activation during the outward journey was correlated with pointing accuracy on a trial-by-trial basis, suggesting that strong engagement allowed for accurate updating of the starting location. Interindividual differences in response consistency were associated with hippocampal and medial prefrontal activation, and the capability to appreciate differences between path layouts could be predicted by activation of the hMT+ complex. Given that, other than the left medial prefrontal cortex, these effects were absent in the control condition, they are unlikely to reflect unspecific memory-related phenomena. In addition, the absence of an overall habituation argues against a possible influence of order effects. We therefore propose that visual path integration is tightly linked to the interplay of self-motion processing in $\mathrm{hMT}+$, higher-level spatial processes in the hippocampus, and spatial working memory in medial prefrontal cortex.

Absolute pointing errors were comparable with previous experiments using similar triangle geometries during virtual (Kearns et al., 2002) and blindfolded (Loomis et al., 1993) navigation. The trend for an overall difference between path integration and control trials may suggest a possible difference in working memory load, but additional analysis revealed this effect to be driven by the $103^{\circ}$ condition. Whereas a difference in working memory load that is restricted to the $103^{\circ}$ trials seems rather unlikely, the amount of self-rotation is a more likely cause. Accurately perceiving self-rotation is difficult when only visual cues are available (Péruch et al., 1997); therefore, the large intermediate rotation in these trials $\left(120^{\circ}\right)$ presumably led to a high uncertainty about its angular value, as indicated by the large SD of the pointing responses.

According to one influential model (Fujita et al., 1993), homing vectors are computed only if required, i.e., after completing an outbound path. Pointing responses during path integration trials may therefore only have been computed in the response phase. In contrast, subjects did not need to compute the pointing response at the end of the trajectory during control trials, because the required pointing direction was already specified at the beginning of each trial. Consequently, the slightly longer reaction times for path integration trials probably reflect additional re- sponse computations, although future experiments need to verify whether the encoding error model in fact applies to visual path integration.

\section{Effects of within-subjects performance variability}

Whenever the hippocampus was strongly activated during the outward journey, participants produced more accurate pointing responses compared with trials with low hippocampal recruitment. Although stronger hippocampal activation during encoding can predict better recall performance in various memory paradigms (Davachi et al., 2001), similar effects did not emerge in the control condition. We therefore interpret the trial-by-trial correspondence as highlighting the importance of the hippocampus for the path integration process.

Path integration requires constant monitoring of distance and direction cues during self-motion. Humans can reliably extract 
self-motion speed from optic flow to discriminate and reproduce traveled distances (Bremmer and Lappe, 1999). Speed-sensitive signals have been recorded in primate areas 7a and MST (Orban et al., 1995; Duffy et al., 2005) and in the hippocampus of the rat (Hirase et al., 1999). Our results strongly indicate that, in humans, the hippocampus receives and integrates such speedrelated signals, which could be used to assess the extent of translational self-motion. Moreover, in rodents, head-direction cells code for the facing direction of the animal's head (Taube, 1998). Although head-direction cells are not generally seen in the rodent hippocampus (but see Leutgeb et al., 2000), it receives directional information (Jeffery et al., 1997). This implies that both direction and distance information converge in the hippocampus to enable updating of position estimates. In accordance with this assumption, we observed lower pointing errors whenever the hippocampal contribution was enhanced. In these trials, integration of distance information from speed-sensitive cells and directional information from head-direction cells was presumably very accurate, which ultimately led to efficient updating of the starting location.

\section{Effects of between-subjects performance variability}

Response consistency

Whereas some participants produced very similar responses across replications of a given path, others varied considerably in their pointing direction. This random error presumably reflects fluctuations in task engagement, and participants with relatively weak hippocampal signals exhibited greater pointing variability, indicating that, in many trials, the hippocampal engagement was not sufficient. As a consequence, the integration of distance and direction became less efficient, which resulted in imprecise updating of the starting location and ultimately in substantial overshooting or undershooting pointing responses.

Random error was also negatively correlated with activation in medial prefrontal cortex. Increased cross-correlation between CA1 place cells and mPFC neurons has been observed in rats using stored information about the starting location in a maze to guide their behavior (Jones and Wilson, 2005). In that study, the synchronization was attenuated during error trials, strongly suggesting that the combined network oscillations reflect the transfer of hippocampal spatial information to an mPFC working memory system. Given that our participants who produced very consistent pointing responses exhibited stronger medial prefrontal activation, we propose the mPFC engagement to reflect constant monitoring and updating of the hippocampal output about changes in position and orientation.

\section{Systematic error}

Signed pointing errors revealed a tendency to produce overshooting responses for small and undershooting responses for large required pointing angles. This systematic error pertains to the individual capacity to encode the outward journey: the higher the uncertainty about the magnitude of translations and rotations, the stronger the tendency to adopt prototypical values (Fujita et al., 1993), which may reflect a bias to perceive outbound paths as isosceles triangles (Kearns et al., 2002) with standard turning angles (i.e., $90^{\circ}$ ). Given that the variability of systematic error has been related to differences in sensory noise and memory decay (Fujita et al., 1993), we suggest the present data to reflect differences in extracting self-motion information from optic flow.

Systematic error was positively correlated with bilateral activation in the middle temporal gyrus, presumably encompassing the human homolog of monkey area MST. In nonhuman primates, dorsal MST contains cells that are can extract the direction of translational heading from optic flow (Duffy et al., 2005). Given the existence of MST in humans with functional properties similar to their monkey counterparts (Dukelow et al., 2001; Huk et al., 2002), the correspondence between activation and systematic error presumably reflects the efficiency of estimating selfmotion. MST neurons in highly proficient subjects precisely extract self-motion speed and direction during translational and rotational movements. These self-motion signals are transmitted to medial temporal lobe structures, including the hippocampus (Duffy et al., 2005), to allow for integrating distance and direction information.

Although it is counterintuitive that good path integrators showed lower hMT + responses than bad path integrators, $\mathrm{hMT}+$ processing is known to be affected by attentional modulation in both macaques (Treue and Maunsell, 1999) and humans (Büchel et al., 1998). Participants who experience difficulties in precisely extracting self-motion from optic flow may devote more attentional resources to the processing in hMT + . As a consequence, the BOLD signal would be upregulated relative to highly proficient subjects. Assuming a limited pool of distributed processing resources, fewer resources would be available to integrate and store distance and direction signals in hippocampoprefrontal loops, which would result in lower hippocampal and prefrontal activation and hence imprecise homing vectors.

In rodents, distance and direction cues already converge in entorhinal grid cells (Hafting et al., 2005), indicating that the hippocampus receives a highly integrated place signal. Whereas grid cells fire immediately in any novel environment and thus mediate a universal spatial metric (McNaughton et al., 2006), place cells are affected by a variety of top-down cognitive processes, including attention (Kentros et al., 2004). Task requirements and attentional fluctuations may therefore have affected hippocampal processing in our subjects, thus modulating the readout of entorhinal signals. In contrast, automatic place computations in entorhinal cortex may be immune to both factors, which would explain the absence of entorhinal effects. Additional analyses on unsmoothed data also failed to reveal any performance-related BOLD responses in this region, thus ruling out a potential confound attributable to spatial smoothing.

In closing, the hippocampal role in representing an observer's spatial position also qualifies previous findings on navigation in large-scale environments (Maguire et al., 1998; Ekstrom et al., 2003; Hartley et al., 2003; Wolbers and Büchel, 2005). Learning and retrieval of complex spatial layouts requires subjects to associate external landmarks with spatial positions. In keeping with existing models (Burgess et al., 2001), we suggest that, during learning, place computations in the hippocampus are combined with parahippocampal/perirhinal landmark representations to construct allocentric descriptions of the environment. During retrieval, external landmarks can reinstantiate place responses in the hippocampus, enabling the observer to plan routes and to correct for accumulating error during path integration. Having established a functional dissociation between the hippocampus, the mPFC, and the hMT + complex, subsequent studies should assess the dynamic interactions between these and other regions that are necessary for computing homing vectors.

\section{References}

Alyan S, McNaughton BL (1999) Hippocampectomized rats are capable of homing by path integration. Behav Neurosci 113:19-31.

Barbas H, Blatt GJ (1995) Topographically specific hippocampal projec- 
tions target functionally distinct prefrontal areas in the rhesus monkey. Hippocampus 5:511-533.

Bremmer F, Lappe M (1999) The use of optical velocities for distance discrimination and reproduction during visually simulated self motion. Exp Brain Res 127:33-42.

Bremmer F, Schlack A, Shah NJ, Zafiris O, Kubischik M, Hoffmann K, Zilles K, Fink GR (2001) Polymodal motion processing in posterior parietal and premotor cortex: a human fMRI study strongly implies equivalencies between humans and monkeys. Neuron 29:287-296.

Bremmer F, Duhamel JR, Ben Hamed S, Graf W (2002) Heading encoding in the macaque ventral intraparietal area (VIP). Eur J Neurosci 16:1554-1568.

Büchel C, Josephs O, Rees G, Turner R, Frith CD, Friston KJ (1998) The functional anatomy of attention to visual motion. A functional MRI study. Brain 121:1281-1294.

Burgess N, Becker S, King JA, O'Keefe J (2001) Memory for events and their spatial context: models and experiments. Philos Trans R Soc Lond B Biol Sci 356:1493-1503.

Collett M, Collett TS (2000) How do insects use path integration for their navigation? Biol Cybern 83:245-259.

Davachi L, Maril A, Wagner AD (2001) When keeping in mind supports later bringing to mind: neural markers of phonological rehearsal predict subsequent remembering. J Cogn Neurosci 13:1059-1070.

Duffy CJ, Page WK, Froehler MT (2005) Posterior cortical processing of self-movement cues: MSTd's role in Papez's circuit for navigation and orientation. In: Head direction cells and the neural mechanisms of spatial orientation (Wiener JM, Taube JS, eds), pp 319-345. Cambridge, MA: MIT.

Dukelow SP, DeSouza JF, Culham JC, van den Berg AV, Menon RS, Vilis T (2001) Distinguishing subregions of the human MT+ complex using visual fields and pursuit eye movements. J Neurophysiol 86:1991-2000.

Eickhoff SB, Stephan KE, Mohlberg H, Grefkes C, Fink GR, Amunts K, Zilles K (2005) A new SPM toolbox for combining probabilistic cytoarchitectonic maps and functional imaging data. NeuroImage 25:1325-1335.

Ekstrom AD, Kahana MJ, Caplan JB, Fields TA, Isham EA, Newman EL, Fried I (2003) Cellular networks underlying human spatial navigation. Nature 425:184-188.

Ellmore TM, McNaughton BL (2004) Human path integration by optic flow. Spat Cogn Comput 4:255-272.

Etienne AS, Jeffery KJ (2004) Path integration in mammals. Hippocampus 14:180-192.

Evans AC, Collins DL, Mills DR, Brown ED, Kelly RL, Peters TM (1993) 3D statistical neuroanatomical models from 305 MRI volumes. Proc IEEE Nuclear Sci Symp Med Imaging 1-3:1813-1817.

Ferino F, Thierry AM, Glowinski J (1987) Anatomical and electrophysiological evidence for a direct projection from Ammon's horn to the medial prefrontal cortex in the rat. Exp Brain Res 65:421-426.

Fujita N, Klatzky RL, Loomis JM, Golledge R (1993) The encoding-error model of pathway completion without vision. Geogr Anal 25:295-314.

Hafting T, Fyhn M, Molden S, Moser MB, Moser EI (2005) Microstructure of a spatial map in the entorhinal cortex. Nature 436:801-806.

Hartley T, Maguire EA, Spiers HJ, Burgess N (2003) The well-worn route and the path less traveled. Distinct neural bases of route following and wayfinding in humans. Neuron 37:877-888.
Hirase H, Czurko HH, Csicsvari J, Buzsaki G (1999) Firing rate and thetaphase coding by hippocampal pyramidal neurons during "space clamping". Eur J Neurosci 11:4373-4380.

Huk AC, Dougherty RF, Heeger DJ (2002) Retinotopy and functional subdivision of human areas MT and MST. J Neurosci 22:7195-7205.

Jeffery KJ, Donnett JG, Burgess N, O’Keefe JM (1997) Directional control of hippocampal place fields. Exp Brain Res 117:131-142.

Jones MW, Wilson MA (2005) Theta rhythms coordinate hippocampalprefrontal interactions in a spatial memory task. PLoS Biol 3:e402.

Kearns MJ, Warren WH, Duchon AP, Tarr MJ (2002) Path integration from optic flow and body senses in a homing task. Perception 31:349-374.

Kentros CG, Agnihotri NT, Streater S, Hawkins RD, Kandel ER (2004) Increased attention to spatial context increases both place field stability and spatial memory. Neuron 42:283-295.

Lee I, Kesner RP (2003) Time-dependent relationship between the dorsal hippocampus and the prefrontal cortex in spatial memory. J Neurosci 23:1517-1523.

Leutgeb S, Ragozzino KE, Mizumori SJ (2000) Convergence of head direction and place information in the CA1 region of hippocampus. Neuroscience 100:11-19.

Loomis JM, Klatzky RL, Golledge RG, Cicinelli JG, Pellegrino JW, Fry PA (1993) Nonvisual navigation by blind and sighted: assessment of path integration ability. J Exp Psychol Gen 122:73-91.

Maguire EA, Burgess N, Donnett JG, Frackowiak RS, Frith CD, O’Keefe J (1998) Knowing where and getting there: a human navigation network. Science 280:921-924.

Maldjian JA, Laurienti PJ, Kraft RA, Burdette JH (2003) An automated method for neuroanatomic and cytoarchitectonic atlas-based interrogation of fMRI data sets. NeuroImage 19:1233-1239.

McNaughton BL, Battaglia FP, Jensen O, Moser EI, Moser MB (2006) Path integration and the neural basis of the "cognitive map". Nat Rev Neurosci 7:663-678.

O'Keefe J, Burgess N, Donnett JG, Jeffery KJ, Maguire EA (1998) Place cells, navigational accuracy, and the human hippocampus. Philos Trans R Soc Lond B Biol Sci 353:1333-1340.

Orban GA, Lagae L, Raiguel S, Xiao D, Maes H (1995) The speed tuning of medial superior temporal (MST) cell responses to optic-flow components. Perception 24:269-285.

Péruch P, May M, Wartenberg F (1997) Homing in virtual environments: effects of field of view and path layout. Perception 26:301-311.

Taube JS (1998) Head direction cells and the neurophysiological basis for a sense of direction. Prog Neurobiol 55:225-256.

Treue S, Maunsell JH (1999) Effects of attention on the processing of motion in macaque middle temporal and medial superior temporal visual cortical areas. J Neurosci 19:7591-7602.

Vann SD, Aggleton JP (2004) Testing the importance of the retrosplenial guidance system: effects of different sized retrosplenial cortex lesions on heading direction and spatial working memory. Behav Brain Res 155:97-108.

Wolbers T, Büchel C (2005) Dissociable retrosplenial and hippocampal contributions to successful formation of survey representations. J Neurosci 25:3333-3340. 